\title{
Oncogenic and drug-sensitive RET mutations in human epithelial ovarian cancer
}

Luyao Guan ${ }^{1}$, Zhang Li ${ }^{2}$ Feifei Xie ${ }^{2}$, Yuzhi Pang ${ }^{2}$, Chenyun Zhang ${ }^{1}$, Haosha Tang ${ }^{1}$, Hao Zhang ${ }^{3}$, Chun Chen ${ }^{1}$, Yaying Zhan ${ }^{1}$, Ting Zhao ${ }^{1}$, Hongyuan Jiang ${ }^{1}$, Xiaona Jia ${ }^{2}$, Yuexiang Wang ${ }^{2 *}$ and Yuan Lu ${ }^{1 *}$

\begin{abstract}
Background: Epithelial ovarian cancer (EOC) is a highly lethal malignancy. Improvement in genetic characterization of EOC patients is required to propose new potential targets, since surgical resection coupled to chemotherapy, presents several limits such as cancer recurrence and drug resistance. Targeted therapies have more efficacy and less toxicity than standard treatments. One of the most relevant cancer-specific actionable targets are protein tyrosine kinases (PTKs) whose role in EOC need to be better investigated.
\end{abstract}

Methods: EOC genomic datasets are retrieved and analyzed. The biological and clinical significance of RET genomic aberrations in ovarian cancer context are investigated by a series of in vitro and in vivo experiments.

Results: Epithelial ovarian cancer sequencing projects identify recurrent genomic RET missense mutations in 1.98\% of patients, ranking as the top-five hit among the 100 receptor tyrosine kinases-encoding genes. RET mutants R693H and A750T show oncogenic transformation properties in NIH3T3 cells. Introduction of the RET mutants into human EOC cells increases RET signaling, cell viability, anchorage-independent cell growth and tumor xenograft growth in nude mice, demonstrating that they are activating mutations. RET mutants significantly enhance the activation of RET and its downstream MAPK and AKT signaling pathway in ovarian cancer cells. Vandetanib, a clinical approved RET inhibitor, inhibits the cell viability and decreases the activation of RET-MAPK signaling pathways in EOC cells expressing oncogenic RET mutants.

Conclusions: The discovery of RET pathogenic variants in the EOC patients, suggests a previously underestimated role for RET in EOC tumorigenesis. The identification of the gain-of-function RET mutations in EOC highlights the potential use of RET in targeted therapy to treat ovarian cancer patients.

Keywords: RET, Ovarian cancer, Vandetanib, Targeted therapy

\footnotetext{
* Correspondence: yxwang76@sibs.ac.cn; yuanlu@fudan.edu.cn

${ }^{2}$ Key Laboratory of Tissue Microenvironment and Tumor, SINH - Changzheng Hospital Joint Center for Translational Medicine, Institutes for Translational Medicine (CAS-SMMU), Shanghai Institute of Nutrition and Health, University of Chinese Academy of Sciences, Chinese Academy of Sciences, 320

Yueyang Rd, Shanghai 200031, People's Republic of China

'Department of Gynecology, Obstetrics and Gynecology Hospital, Fudan University Shanghai, 419 Fangxie Rd, Shanghai 200011, People's Republic of China

Full list of author information is available at the end of the article
}

\section{Background}

Ovarian cancer remains the most deadly gynecological malignancy due to the advanced stage at which patients are diagnosed. The number of new cases in 2019 is estimated to be 22,530, and the estimated number of deaths is approximately 13,980 globally [1]. Cytoreductive surgery combined with platinum-based chemotherapy has been the standard of care for advanced ovarian cancer for the last 35 years [2]. Although patients are sensitive to platinum initially, they

C C The Author(s). 2020 Open Access This article is licensed under a Creative Commons Attribution 4.0 International License, which permits use, sharing, adaptation, distribution and reproduction in any medium or format, as long as you give appropriate credit to the original author(s) and the source, provide a link to the Creative Commons licence, and indicate if changes were made. The images or other third party material in this article are included in the article's Creative Commons licence, unless indicated otherwise in a credit line to the material. If material is not included in the article's Creative Commons licence and your intended use is not permitted by statutory regulation or exceeds the permitted use, you will need to obtain permission directly from the copyright holder. To view a copy of this licence, visit http://creativecommons.org/licenses/by/4.0/. The Creative Commons Public Domain Dedication waiver (http://creativecommons.org/publicdomain/zero/1.0/) applies to the data made available in this article, unless otherwise stated in a credit line to the data. 
develop platinum resistance after multiple relapses, and the effectiveness of second-line chemotherapy is limited [3]. In addition, increasing evidence has shown that ovarian cancer should not be regarded as a single entity, as different subtypes have distinct clinical, histological, and molecular features [4]. Compared with empirical therapy, molecularly targeted therapy might be a more effective and less toxic therapy for ovarian cancer patients.

PARP inhibitors (PARPi) and antiangiogenic agents are the two main kinds of molecularly targeted drugs introduced as frontline therapy for ovarian cancer [4]. PARPi demonstrated significant promotion of progression-free survival (PFS) and the latest reported overall survival (OS) period when used as a maintenance therapy in recurrent ovarian cancer patients; the benefits are mainly shown in platinumsensitive patients or patients with $B R C A$ mutations [5], and patients without HRD (homologous recombination deficiency) may not benefit from PARPi according to the "synthetic lethal" theory. Antiangiogenic agents mainly include monoclonal antibodies such as bevacizumab targeting vascular endothelial growth factor (VEGF) and tyrosine kinase inhibitors (TKIs) targeting VEGF receptor (VEGFR). Antiangiogenic therapies have been integrated into the treatment of ovarian cancer patients per the recommended guidelines, but the OS benefits need to be identified considering the cost-effectiveness and toxicity. There have been no licensed targeted agents for ovarian cancer since bevacizumab was approved in 2014 [6], which encouraged us to explore whether there are other targets for the treatment of ovarian cancer patients.

Protein tyrosine kinases (PTKs) genes are a major kind of oncogene divided into transmembrane receptor tyrosine kinases (RTKs) and cytoplasmic nonreceptor tyrosine kinases (NRTKs) genes. PTKs genes are involved in survival, proliferation, invasion, and angiogenesis in many cancers, making them potential therapeutic targets in cancer treatments. With the deeper understanding of kinases and the faster development of pharmaceuticals, there were 19 kinase inhibitors approved in 4 years (from 2011 to 2015, [7]). Taking gefitinib as an example, it was licensed to treat non-smallcell lung cancer (NSCLC) patients with epidermal growth factor receptor (EGFR) mutations and yielded a significant PFS benefit (10.4 versus 5.5 months) compared with the chemotherapy group [8]. These encouraging facts led us to explore the oncogenic role of PTKs in ovarian cancer.

RET (rearranged during transfection) is a single transmembrane RTK that consists of an extracellular domain containing four cadherin-like domains and a cysteinerich domain, a transmembrane domain, and an intracellular kinase domain [9] (Fig. 1a). As a typical RTK, mutation, rearrangement, and aberrant expression of the $R E T$ gene induces the autophosphorylation of RET, and then phosphorylated RET phosphorylates downstream signaling pathways to drive various cancers, such as hereditary and sporadic medullary thyroid carcinoma (MTC) [10, 11], papillary thyroid cancers [12], and NSCLC [13]. Vandetanib, a TKI (tyrosine kinase inhibitors) with inhibitory activity against RET, has been approved for the treatment of patients with locally advanced and metastatic MTC whose pathogenesis mainly comes from $R E T$ mutations [14]. A phase III clinical trial showed that MTC patients with RET mutations benefited more from vandetanib [15], which suggested vandetanib might serve as a therapeutic choice for patients with RET alterations. We studied the oncogenic functions of $R E T$ mutations and tested the therapeutic effects of vandetanib in ovarian cancer.

As epithelial ovarian cancer (EOC) accounts for over 95\% of ovarian cancer [16], we focused on EOC in this study. First, we collected and analyzed data on RET mutations in EOC from genomic databases and found the oncogenic effects of RET mutations on promoting cell viability and colony formation of ovarian cancer cells in vitro and accelerating tumor xenograft growth of ovarian cancer in vivo. Next, we demonstrated that the kinase inhibitor vandetanib could effectively inhibit the viability of ovarian cancer cells with RET mutations. The RET-MAPK signaling pathway might be the main mechanism by which RET mutants promote the development of ovarian cancer. Our study identified the oncogenic role of $R E T$ mutations in ovarian cancer and provided a potential treatment option for ovarian cancer patients with RET mutations.

\section{Methods \\ Identification of potential active mutations}

To identify RET mutations in ovarian cancer, we searched widely used genomic databases, including TCGA (The Cancer Genome Atlas), COSMIC (Catalogue of Somatic Mutations in Cancer), ICGC (International Cancer Genome Consortium), and CCLE (Broad Institute Cancer Cell Line Encyclopedia). To filter potential active mutations, we set two criteria: 1 ) the mutations predicted to be "possibly damaging" or "probably damaging" in PolyPhen-2 or predicted to be "damaging" in SIFT (Sorting Intolerant from Tolerant) were included, and 2) the amino acid substitutions presented in the 1000 Genomes Project were excluded.

\section{Construction of mutant vectors and packaging lentivirus}

The PCDH3.1-RET51-WT (wild-type) plasmid was a generous gift from Professor Lois M. Mulligan. This plasmid was digested with NOT1 (\#R3189S, New England Biolabs) and NheI (\#R3131S, New England Biolabs) restriction endonucleases to obtain the coding sequences (CDS) of RET. The pCDH-CMV-MCS-EF1-Neo plasmid (\#CD514B-1, System biosciences) was used as the empty vector $(\mathrm{EV})$ and was treated in the same way, and then the products were ligated using T4 DNA 
A

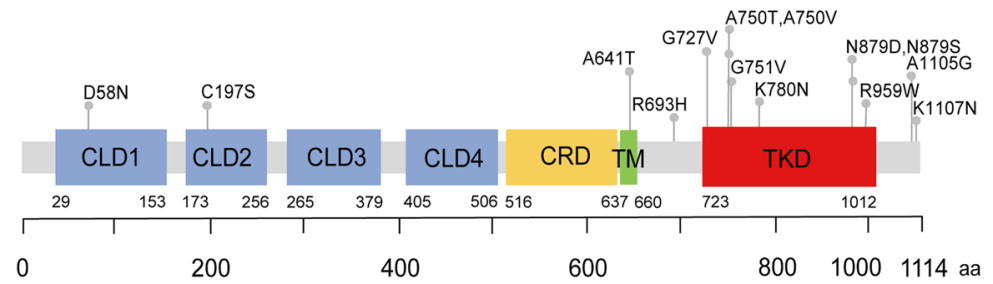

B

Genes with a mutation frequency ranking top 5 of 100 PTKs genes

\begin{tabular}{lccc}
\hline PTK genes & Mutation frequency $(\%)$ & $\begin{array}{c}\text { Inhibitors approved } \\
\text { by FDA }\end{array}$ & Reference \\
\hline MST1R & 2.98 & - & Mayer S et al., 2015 \\
INSR & 2.15 & - & Haeusler RA et al., 2018 \\
RET & 1.98 & Vandetanib & - \\
PTK7 & 1.98 & - & Damelin M et al., 2017 \\
PDGFRB & 1.98 & Sunitinib & Weissmueller S et al., 2014
\end{tabular}

C

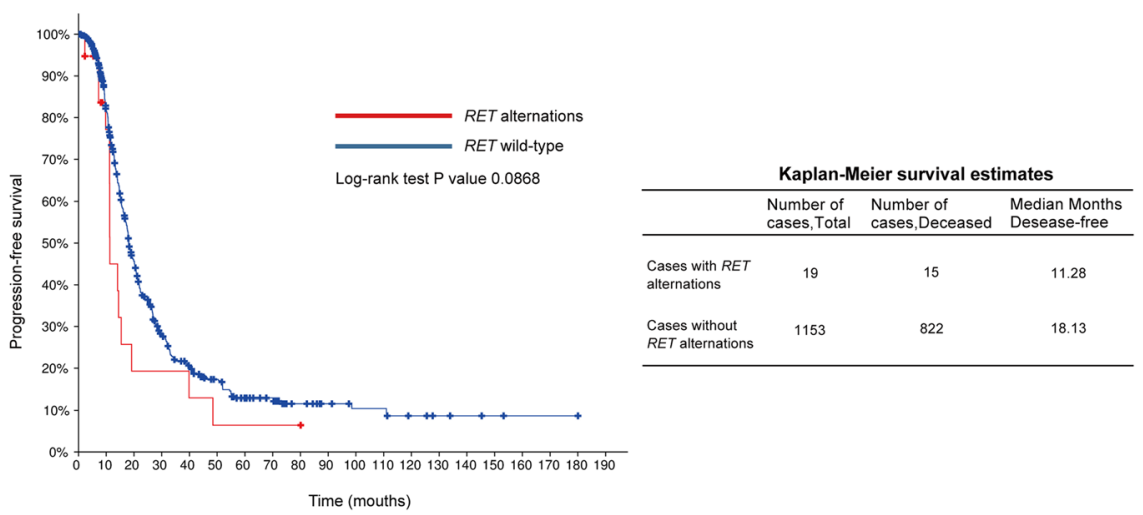

Fig. 1 RET alterations in ovarian cancer. a Missense mutations in the CDS (coding sequence) of RET from the TCGA, COSMIC, ICGC and CCLE databases are marked over the affected protein domains. CLD, cadherin-like domain; CRD, cysteine-rich domain; TM, transmembrane domain; TKD, tyrosine kinase domain. b Genes with a mutation frequency ranking in the top 5 of 100 PTKs in ovarian cancer are listed, and the mutation frequency, FDA approved inhibitors and representative reference are also shown. c Kaplan-Meier plot of the progression-free survival of ovarian cancer patients based on RET alterations from the TCGA database

Ligase (M0202S, NEB) to gain pCDH-RET51-WT plasmid (WT). RET mutants were constructed by site-directed mutagenesis of the pCDH-RET51-WT plasmid using the QuikChange Lightning Site-Directed Mutagenesis Kit (\#210518, Agilent). The constructs with RET mutations were confirmed by sequencing. The lentivirus particles containing EV, WT, RET mutants were generated as described [17].

\section{Cell culture and transfection}

HEK293T cells (\#ACS-4500), NIH3T3 cells (\#CRL-1658) and pancreatic ductal epithelia cell line MiaPaCa-2 (\#CRL1420) were purchased from ATCC. The EOC cell line A2780 and SKOV3 was obtained from the Cell Bank of the Chinese Academy of Sciences (Shanghai, China) and OVK18 was purchased from RIKEN (RCB1903). All cells were validated by short-tandem-repeat (STR) DNA fingerprinting. HEK293T cells, NIH3T3 cells, A2780 cells and SKOV3 cells were supplemented with RPMI 1640 (\#11875093, Thermo Fisher Scientific). MiaPaCa-2 were supplemented with DMEM/F-12 (\#11330057, Thermo Fisher Scientific). OVK18 cells were cultured with MEM (\#11095080, Thermo Fisher Scientific). All above media contain 10\% fetal bovine serum (\#10099141, Thermo Fisher Scientific) and streptomycin/ penicillin and maintained in a 5\% CO2 humidified atmosphere at $37^{\circ} \mathrm{C}$. All cell lines were routinely tested for microbial contamination (including mycoplasma). None of the cell lines in this study appears in the misidentified cell line list kept by the ICLAC. 
For transient transfection, $1 \mu \mathrm{g}$ constructed plasmids were transfected into HEK293T cells using Lipofectamine 2000 (\#11668019, Thermo Fisher Scientific) according to the manufacturer's instructions. For stable transfection, cells were transduced with RET mutants when cell confluence reached $50 \%$ and were selected with $2 \mu \mathrm{g} / \mathrm{ml}$ puromycin for 4 days before use.

\section{Western blot analysis}

Cells were lysed with lysis buffer (1\% NP-40, $50 \mathrm{mM}$ Tris-HCl $\mathrm{pH}$ 8.0, $100 \mathrm{mM}$ sodium fluoride, $30 \mathrm{mM}$ sodium pyrophosphate, $2 \mathrm{mM}$ sodium molybdate, $5 \mathrm{mM}$ ethylenediaminetetraacetic acid and $2 \mathrm{mM}$ sodium orthovanadate) containing protease inhibitors (leupeptin, aprotinin, and phenylmethylsulfonyl fluoride). Lysates were quantified using the Quick Start Bradford 1× Dye Reagent (\#5000205, Bio-Rad). Equal amounts of protein were separated on $10 \%$ SDSpolyacrylamide gels and then transferred to nitrocellulose membranes. The membranes were incubated with primary antibodies at $4{ }^{\circ} \mathrm{C}$ overnight and blocked with HRPconjugated secondary antibody at room temperature for $1 \mathrm{~h}$. The signals were detected using a chemiluminescence kit (Immobilon Western, Millipore Corporation, MA). Quantification of band intensity was analyzed by Image Quant TL 8.1 software (GE Healthcare).

\section{Antibodies and chemicals}

The primary antibodies used in this study included antiphospho-RET (Tyr905, \#3221), anti-RET (\#14556), antiphospho-ERK (Thr202/Tyr204, \#9101S), anti-ERK (\#9102), anti-phospho-AKT (Ser473, \#9271) and anti-AKT (\#9272). They were purchased from Cell Signaling Technology. AntiGAPDH (\# G8795) was purchased from sigma.

Vandetanib was purchased from Selleck Chemicals and diluted with DMSO to 6 concentrations: 0, 500, 750, 1000, 2000, and $5000 \mathrm{nM}$. For vandetanib inhibition test, A2780 cells transfected with RET mutants were cultured with different concentrations of vandetanib for $72 \mathrm{~h}$. For western blotting, A2780 cells transfected with EV, WT or RET mutants were treated with $500 \mathrm{nM}$ vandetanib for $4 \mathrm{~h}$ before harvesting.

\section{Cell Titer-Glo (CTG) assay}

A2780 cells (2000 cells per well) transfected with EV, WT or RET mutants were seeded in 96-well plates in triplicate. Cell viability was determined using CTG (\#G7572, Promega) according to the manufacturer's instructions. The relative cell viability was calculated as the cell viability at 96 $h$ relative to that at $24 \mathrm{~h}$. The inhibition effect of vandetanib was calculated as the cell viability of cells treated with concentrations of 500, 750, 1000, 2000, and $5000 \mathrm{nM}$ relative to that of cells treated with $0 \mathrm{nM}$ at $96 \mathrm{~h}$. The experiments were repeated three times.

\section{Soft agar assay and plate clone formation assay}

To examine anchorage-independent growth, NIH3T3 cells $\left(2 \times 10^{5}\right.$ cells per well) transfected with EV, WT or RET mutants were suspended in $0.35 \%$ agar (\#214220, BD Difco) containing RPMI 1640 medium with 10\% fetal bovine serum and penicillin/streptomycin and seeded on $0.7 \%$ agar with the same complete media in 6-well plates. Cells were allowed to grow for 5 weeks and stained with methyl thiazol tetrazolium (\#M5655, Sigma-Aldrich) for $3 \mathrm{~h}$. The number of colonies was counted manually. This assay was performed in triplicate.

For plate clone formation assay, A2780 cells (500 cells per well) transfected with EV, WT or RET mutants were seeded in 6-well plates in triplicate for 14 days. Cells were fixed with methanol for $15 \mathrm{~min}$ and stained with crystal violet for $30 \mathrm{~min}$. The number of colonies was counted manually. This experiment was performed three times.

\section{Xenograft assays}

The xenograft experiment was approved by the Animal Ethics Committee of Fudan University. Five-week-old female nude mice (BALB/c nude, SLAC, Shanghai, China) were randomly divided into 4 groups (4 mice per group) and inoculated subcutaneously into the left flank with A2780 cells $\left(3 \times 10^{6}\right.$ cells per mice $)$ transfected with EV, WT or RET mutants in $50 \mu \mathrm{l}$ PBS and an equal volume of Matrigel (\#356234, Corning). All mice were sacrificed 3 weeks later, and the tumors were removed to measure volume and weight. Tumor volume was calculated using the formula: Tumor volume $=$ length $\times$ width $^{2} / 2$. This experiment was repeated three times.

\section{Statistical analysis}

Statistical analysis and graph generation were performed using GraphPad Prism 7.0. Unpaired two-tailed Student's ttest was used to evaluate the significance of differences between two groups. A $P$-value $<0.05$ was considered statistically significant.

\section{Results}

\section{Recurrent RET mutations identified in ovarian cancer patients}

To search for PTKs-encoding gene mutations with oncogenic potential in ovarian cancer, we collected and analyzed genome sequencing data from the TCGA $(n=605)$ and found that the genes with a mutation frequency ranking in the top 5 of 100 PTK genes (Fig. 1a; Additional file 1: Supplementary Figure 1 and Additional file 2: Supplementary Table 1) in epithelial ovarian cancer were MST1R, INSR, RET, PDGFRB, and PTK7,among which RET (reference transcript, NM_02097; reference protein, NP_066124) gene mutations had not been studied in ovarian cancer patients and had approved inhibitors (Fig. 1b; Additional file 2: Supplementary Table 1). Endogenous RET is expressed in 
epithelial ovary cancer at both RNA and protein levels (Additional file 1: Supplementary Figure 2A-2B).

Patients with $R E T$ gene mutations were identified in 12 out of 605 (1.98\%) ovarian cancer patients (Additional file 2: Supplementary Table 2), and those with $R E T$ alterations had shorter progression-free survival than those without $R E T$ alterations (11.27 versus 18.13 months; Fig. 1c), which indicated that $R E T$ alterations play a role in the progression of ovarian cancer. To obtain a comprehensive view of RET mutations in ovarian cancer, we also collected $R E T$ mutations from other sequencing databases, including COSMIC, ICGC and CCLE. There were 22 missense mutations in total. To select potential activating RET mutations among these mutations, we used Polyphen2 [18] and SIFT [19], two commonly used prediction algorithms, to predict whether specific amino acid substitutions of RET would affect protein function based on the sequence homology and structure of protein. Ultimately, 14 missense mutations were further studied, as other substitutions were either predicted to be benign according to the scores of PolyPhen-2 and SIFT (R79Q, R114H, R205S, G248S, A342G, T636M, T1085A, and A680T) or presented in the 1000 Genomes Project (A680T) (Additional file 2: Supplementary Table 3).

\section{Oncogenic transformation properties of RET mutations}

To study the oncogenic potentials of the 14 mutations, we transiently transfected plasmids expressing these mutations into HEK293T cells and detected their effects on the phosphorylation of RET. C634R was used as the positive control of the oncogenic RET mutation [20, 21]. R693H, A750T and C634R mutants significantly increased the ratio of phosphorylated RET to total RET compared with that of cells transfected with wild type RET (WT) or empty vector (EV) (Fig. 2a and b), which indicated that these two mutations were able to activate RET. A641T seemed not an active mutation because it is not able to activate RET in HEK293T cells or NIH3T3 cells (Additional file 1: Supplementary Figure 3A-3C).

To further identify the effects of R693H and A750T on anchorage-independent growth, we used the NIH3T3 mouse embryo fibroblast cell line as a model that is commonly used to evaluate the transforming ability of oncogenes [22]. Additionally, NIH3T3 cells do not express endogenous RET [21] (Additional file 1: Supplementary Figure 2B). We constructed lentiviral vectors encoding RET mutants and transduced them into NIH3T3 cells. R693H and A750T significantly increased the phosphorylation of RET in the Western blot, demonstrating that RET was activated in NIH3T3 cells (Fig. $2 \mathrm{c}$ and $\mathrm{d}$ ). The colony formation assay showed that NIH3T3 cells transduced with EV and WT vectors formed few colonies in soft agar, while R693H and A750T vectors significantly increased the number of colonies (Fig. 2e and f), which demonstrated that $\mathrm{R} 693 \mathrm{H}$ and A750T promote anchorage-independent growth and that both of them are gain-of-function mutations.
RET mutations activate RET signals and promote ovarian cancer growth in vitro and in vivo

To further evaluate the oncogenic functions of R693H and A750 T in ovarian cancers, we transduced lentiviral vectors expressing these mutants or WT or C634R mutant (positive control [23]) into an EOC cell line A2780. The A2780 cell line is a widely used, low-RET-expressing cell line in the CCLE. Western blot analysis showed that the R693H or A750T mutant significantly elevated the phosphorylation of RET. As the MAPK and AKT signaling pathways are the main downstream signal transduction pathway of RET, we detected the ratio of phosphorylated ERK to total ERK and phosphorylated AKT to total AKT to assess the activation of downstream signal pathways and found that cells expressing the R693H or A750T mutant had higher ERK phosphorylation than cells expressing WT (Fig. 3a-c), which confirmed that both mutations promote the activation of the MAPK and AKT signaling pathways.

A CTG assay was performed to investigate the role of $\mathrm{R} 693 \mathrm{H}$ and $\mathrm{A} 750 \mathrm{~T}$ mutations in promoting cell viability. We found that viability was higher in the $\mathrm{R} 693 \mathrm{H}$ or $\mathrm{A} 750 \mathrm{~T}$ mutant-expressing A2780 cells than in the WT-expressing cells (Fig. 3d). A plate colony formation assay showed similar results: the number of colonies was higher in A2780 cells transduced with $\mathrm{R} 693 \mathrm{H}$ or A750T mutant than in cells transduced with WT (Fig. 3e-f). Next, we investigated the oncogenic role of RET mutations in the growth of tumor xenografts in nude mice and found that WT and EV barely increased the growth of A2780 cells. In contrast, A2780 cells expressing the $\mathrm{R} 693 \mathrm{H}$ or A750T mutant significantly increased the volume and weight of tumors compared with those of WT (Fig. 3g-i).

In conclusion, $\mathrm{R} 693 \mathrm{H}$ and $\mathrm{A} 750 \mathrm{~T}$ mutants of RET enhance the signal transduction of RET, the cell viability and colony formation of cells, and the growth of tumor xenografts of ovarian cancer.

\section{Vandetanib inhibits the viability and reduces the cell signaling of ovarian cancer cells expressing $\mathrm{R} 693 \mathrm{H}$ or A750T mutants}

Vandetanib is an inhibitor with strong activity against RET, EGFR, and VEGFR, which demonstrated more benefit in MTC patients with RET mutations than in patients without $R E T$ mutations. To investigate whether vandetanib has the potential to be applied in ovarian cancer patients with RET mutations, we tested the inhibitory effects of vandetanib on A2780 cells expressing the R693H or A750T mutant. The results showed that vandetanib dramatically decreases cell viability at a concentration of $500 \mathrm{nM}$ and revealed a dosedependent relationship between vandetanib and viability (Fig. 4a). To determine the molecular mechanisms of the inhibitory effects, western blotting was performed and demonstrated that vandetanib reduces the phosphorylation of RET and ERK in A2780 cells expressing the R693H or A750T 
A

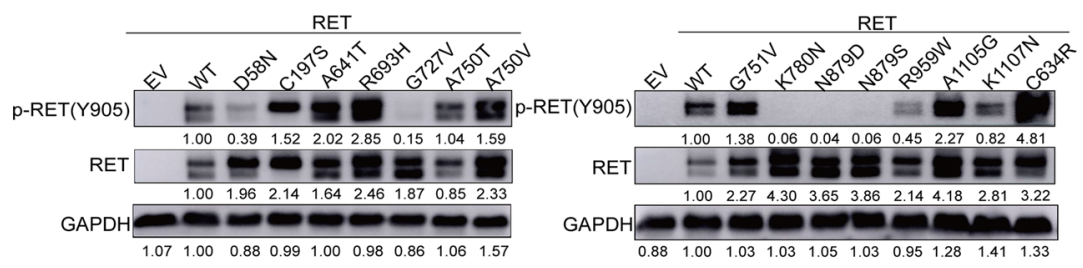

B

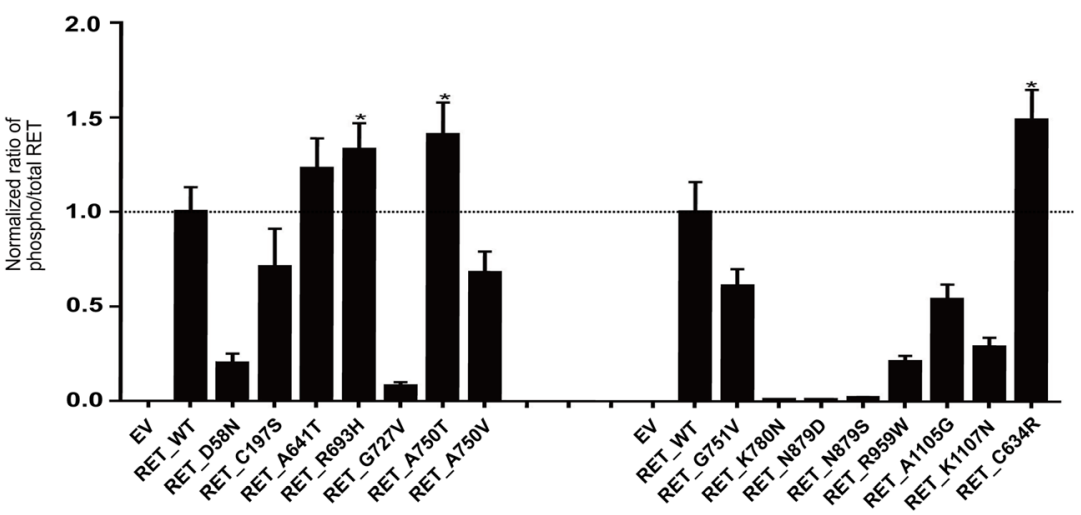

C

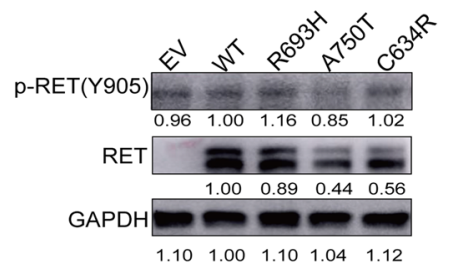

E

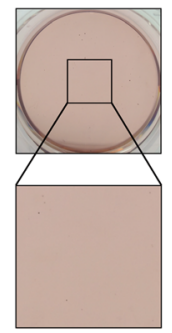

EV

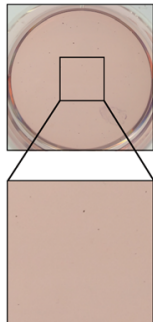

WT

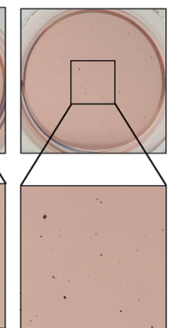

$\mathrm{R} 693 \mathrm{H}$
D

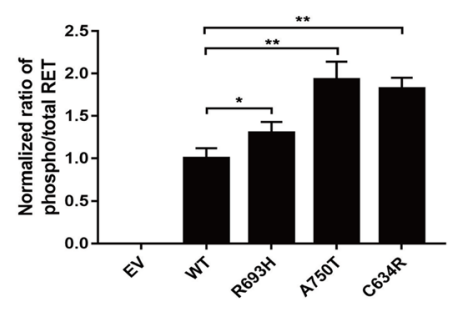

F

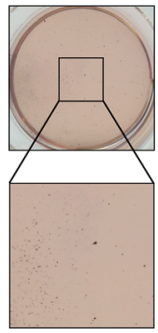

A750T

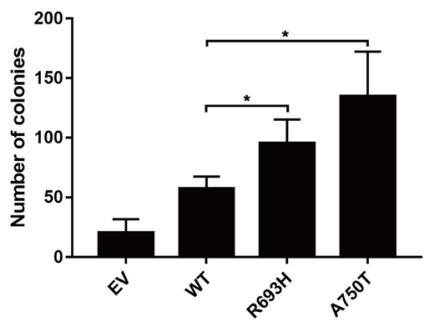

Fig. 2 Oncogenic transformation properties of RET mutations. a HEK293T cells were transfected transiently with empty vector (EV), RET wild-type (WT) or RET mutants (14 potential active mutations and C634R mutant as the positive control). The lysates were analyzed by western blotting with anti-phospho RET (Y905) and anti-RET antibodies. b Bar graphs demonstrated the quantification of western blotting bands in (a), normalized to WT control. c NIH3T3 cells were transfected stably with empty vector (EV), RET wild-type (WT) or RET mutants (R693H and A750T) viruses. The lysates were analyzed by western blotting with anti-phospho RET (Y905) and anti-RET antibodies. $\mathbf{d}$ Bar graphs showed the quantification of western blotting bands in (c), normalized to WT control. e and $\mathbf{f}$ RET mutants promote the anchorage-independent growth of NIH3T3 cells. $\mathrm{NIH} 3 \mathrm{T3}$ cells stably transfected with EV, WT, or RET mutants were seeded in soft agar in triplicate for 5 weeks and stained with methyl thiazol tetrazolium (MTT), representative plates $(\mathbf{f})$ and the number of colonies $(\mathbf{f})$ are shown. All of the above results represent three repeated experiments ${ }^{*}, P<0.05$ compared to WT

mutant (Fig. 4b-d), which was consistent with the conclusion that the $\mathrm{R} 693 \mathrm{H}$ or A750T mutant enhances RET signal transduction. We inferred that vandetanib has the potential to treat EOC patients with RET gain-of-functional mutations.

\section{Discussion}

Debulking surgery combined with platinum-based chemotherapy as an empirical treatment for advanced ovarian cancer has been used for the last 35 years, while the 5-year survival of late-stage ovarian cancer is still < 


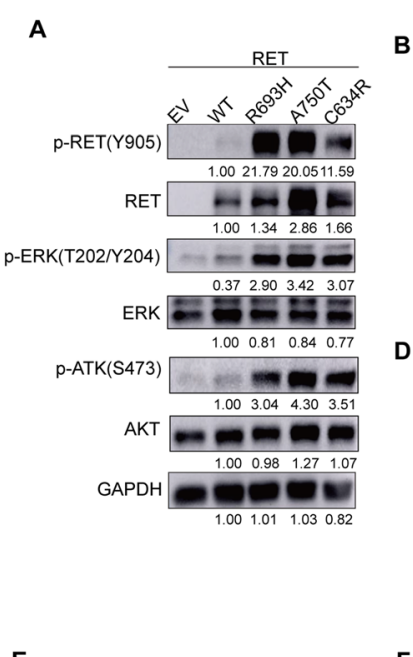

E

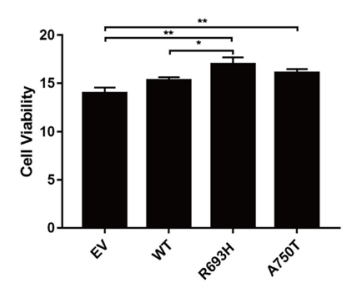

H

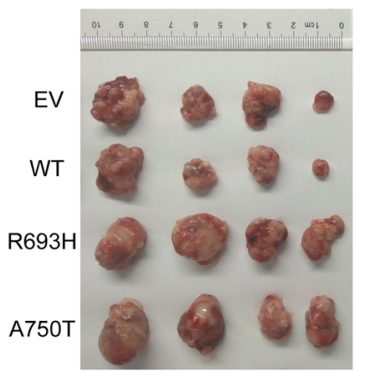

$\mathbf{F}$

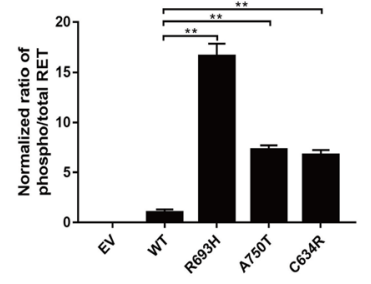

C
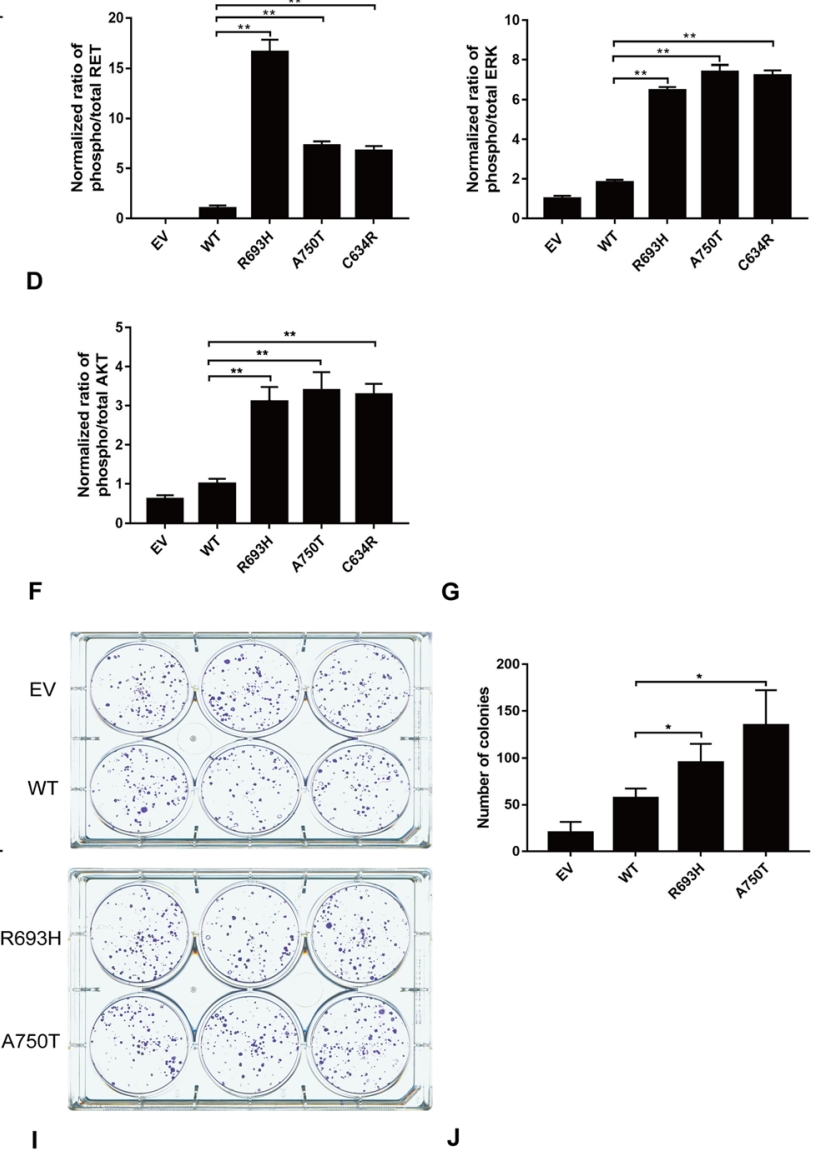

G
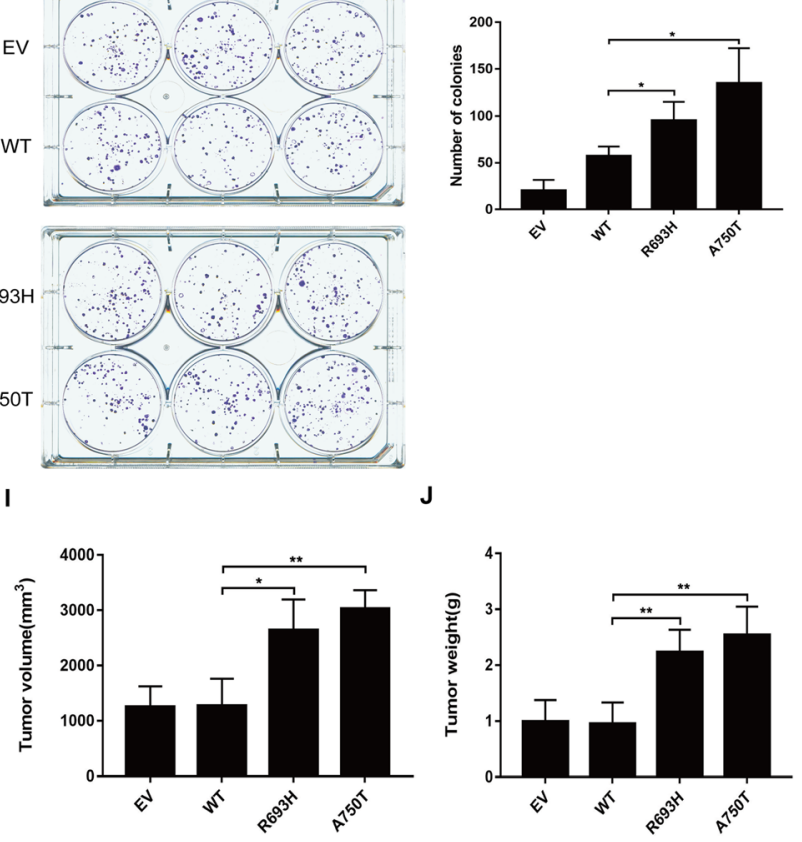

Fig. 3 Oncogenic potential of RET mutants in vitro and in vivo. a Lysates from epithelial ovarian cancer cell (EOC) A2780 transduced with EV or WT or mutants were analyzed by immunoblot with anti-phospho RET (Y905), anti-RET antibody, anti-phospho ERK (T202/204), anti-ERK antibody, anti-phospho AKT (S473), and anti-AKT antibody (b-d) Bar graphs showing quantification of western blot bands in (a), normalized to WT control. e R693H and A750T mutants increase the cell viability of A2780 cells. A2780 cells stably expressing EV, RET WT or RET mutants grew on 96-well plates in triplicate for $72 \mathrm{~h}$, and the viability was measured by CTG assay. $\mathbf{f}$ and $\mathbf{g}$ A2780 cells transduced with EV, RET WT or mutants were seeded in 6-well plates (500 cells per well) in triplicate for 2 weeks and stained with MTT. Representative plates (f) and number of colonies are presented $(\mathbf{g}) \cdot \mathbf{h}-\mathbf{j}$ RET mutants promote the growth of ovarian cancer xenografts in nude mice. Representative pictures (h), tumor volumes (i) and tumor weights (j) are shown. ${ }^{*}, P<0.05$ compared to WT. ${ }^{*}, P<0.01$ compared to WT

$30 \%$ [1]. Both the lack of effective treatments after the development of platinum resistance and the heterogeneity of ovarian cancer pushed us to explore the potential of targeted therapy for ovarian cancer. Additionally, targeted therapy has the advantage of improving therapeutic effects and reducing therapeutic toxicity [23]. PARPi and bevacizumab are two primary molecularly targeted agents in ovarian cancer. Both of these agents demonstrated PFS benefit, and PARPi also showed OS benefit in platinum-sensitive recurrent ovarian cancer patients [5]; however, they both lack definite biomarkers to predict therapeutic outcome, which suggests that neither could represent virtually personalized therapy [24]. 
A

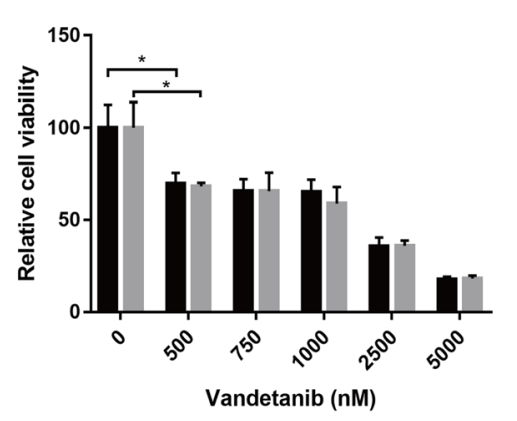

B

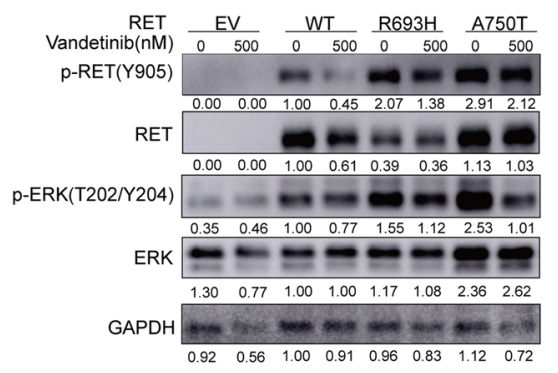

$\mathrm{R} 693 \mathrm{H}$

A750T

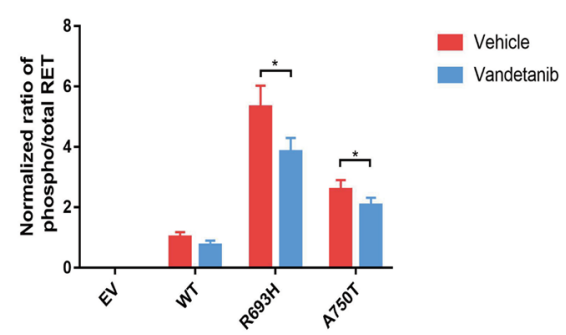

D

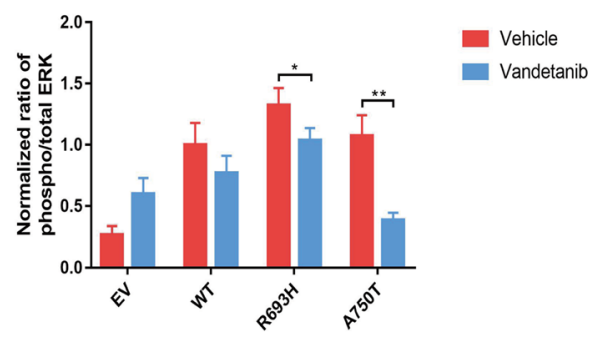

Fig. 4 Vandetanib inhibits the viability and cell signaling of RET in EOC cells expressing RET mutants. a Drug sensitivity of RET mutants in the CTG assay. A2780 cells transduced with RET R693H or A750T were seeded in 96-well plates in triplicate and treated with 0, 500, 750, 1000, 2500, or $5000 \mathrm{nM}$ vandetanib at $24 \mathrm{~h}$ and $72 \mathrm{~h}$ after plating. The inhibition effects of vandetanib are shown in the bar graph and normalized to the WT control. b Vandetanib strongly inhibits the phosphorylation of RET and ERK in A2780 cells with RET mutations. A2780 cells expressing either RET R693H or A750T mutant were treated with $500 \mathrm{nM}$ vandetanib for $4 \mathrm{~h}$ before harvesting, and the western blotting results are shown in (b). $\mathbf{c}$ and d Bar graphs showing quantification of western blotting bands in (b), normalized to WT control. The results represent three repeated experiments *, $P<0.05$ compared to WT. ${ }^{*}, P<0.01$ compared to WT

PTKs are the main targets in the targeted treatment of cancer. The first approved targeted agent was trastuzumab to treat HER2-positive breast cancer patients in 1998 [25]. Since then, an increasing number of PTKs have been recognized as therapeutic targets, such as the "miracle" drug imatinib which targets BCR-ABL. Its emergence improved the complete cytogenetic response of Philadelphia chromosome-positive chronic myelogenous leukemia by almost 50\% [26]. The remarkable effects and the emergence of PTKs inhibitors provides us with an opportunity to study whether PTKs could be regarded as potential targets in ovarian cancer.

In our study, we identified the genes with a mutation frequency ranking in the top 5 of 100 PTK genes in
EOC, namely, MST1R, INSR, RET, PDGFRB, and PTK7, among which we studied the overlooked oncogenic role of RET in ovarian cancer. Our results showed that RET $\mathrm{R} 693 \mathrm{H}$ and A750T mutants could promote the viability, colony formation and activation of the RET-MAPK and RET-AKT signaling pathway in ovarian cancer, and the RET inhibitor vandetanib could significantly decrease cell viability and signal transduction at $500 \mathrm{nM}$. Our study provides a good starting point for the application of RET inhibitors in EOC patients with RET mutations.

The total RET and total ERK protein levels were different among mutants as shown in Figs. 2a and 3a, which may be explained by the different lentiviral infection efficiency (due to different virus titers) among 
mutants. The phosphorylated protein and total protein were loaded proportionally in the same western blot experiment. Hence the ratio of phosphorylated protein/ total protein was used to prove the activation effects of various $R E T$ mutations.

R693H and A750T are located in the juxtamembrane (JM) region and intracellular kinase domain, respectively. Mutations in the kinase domain were commonly reported in MTC, including the most common RET mutation M918T, which was associated with more severe disease phenotypes of MTC [27]. The oncogenic mutations in this domain may induce conformational changes in proteins, which decrease autoinhibition, increase kinase activity and ATP binding, generate a better intermolecular substrate [28, 29], and then cause the autophosphorylation of RET to induce cancer. There are some publications about the polymorphism in the JM region of RET. The G691S RET polymorphism was reported to enhance the response of RET to glial cell linederived neurotrophic factor (GDNF) and was correlated with the aggressive phenotype of pancreatic cancers [30] and cutaneous malignant melanomas [31]. This substitution is close to the RET R693H mutant we studied, which reflects the importance of variations in this domain in cancer, while the allosteric mechanisms are not yet known. Interestingly, there was a decrease in the level of RET protein after vandetanib treatment, especially in the WT-expressing cells (Fig. 4b). How the vandetanib decreases the expression of total WT RET warrants further investigation.

There are some potential limitations to our study: 1) Compared with other targets, the mutation frequency of PTKs genes, including $R E T$, is low, while according to the conception of precision therapy, we believe that patients with $R E T$ mutations would benefit from the personalized treatment strategy targeting RET. International cooperation and case sharing will facilitate the application of PTKs inhibitors. 2) Most currently used PTKs inhibitors, including vandetanib, are multikinase inhibitors, which might influence the therapeutic specificity and thus weaken the curative efficacy. The development of next-generation selective RET inhibitors such as BLU-667 [32] and LOXO-292 [33] may contribute to the precision therapy of patients with $R E T$-mutant ovarian cancer. Taking BLU-667 as an example, it was reported to have 88-fold more potency against RET than VEGFR [34], and it has already demonstrated clinical benefits in RET-altered thyroid cancer and lung cancer patients in early stage clinical trials $[35,36]$.

It has been only 5 years since the first FDA-approved targeted medicine emerged in ovarian cancer. To date, the effects of PTKs inhibitors in ovarian cancer patients in early-stage clinical trials have been modest compared with the effects of therapies targeting oncogenic drivers in other solid tumors [37]. The explanation might come from the lack of patient selection in the trials. In addition, the heterogeneity creates a more complex landscape of ovarian cancer, so combination therapy with other chemotherapies or targeted drugs might be helpful, and the determination of a precise treatment based on the dynamic characteristics of the genome of ovarian cancer would also be beneficial [38].

The application of next-generation genetic sequencing technology, the unceasing exploration of genetics, the expansion of noninvasive biomarker detection technology [39, 40], and the dramatic development of the pharmaceutical industry will accelerate the development of precision medicine for ovarian cancer and eventually reduce the morbidity and mortality of ovarian cancer patients. We believe that, in the future, therapies targeting RET will prolong survival while not influencing the quality of life of patients with RET-mutant ovarian cancer.

\section{Conclusions}

The discovery of RET pathogenic variants in the EOC patients, suggests a previously underestimated role for RET in EOC tumorigenesis. The identification of the gain-of-function RET mutations in EOC highlights the potential use of RET in targeted therapy to treat ovarian cancer patients.

\section{Supplementary information}

Supplementary information accompanies this paper at https://doi.org/10. 1186/s13046-020-01557-3.

\begin{abstract}
Additional file 1: Supplementary Figure 1. PTKs-encoding genes. Supplementary Figure 2. Endogenous RET expression in human ovarian cancer cells. (A) The RNA-seq data from epithelial ovary carcinoma patients from the TCGA program (TCGA Pan Can Atlas study: $n=585$; TCGA Provisional study; $n=606$ ) were retrieved and analyzed. Endogenous RET is expressed in epithelial ovary carcinoma at RNA level. (B) Lysates from two epithelial ovarian cancer cell lines, SKOV3 and OVK18, were analyzed by western blot with anti-RET antibody and GAPDH antibody. The pancreatic ductal epithelia cell line MiaPaCa-2 and NIH3T3 cell line were positive and negative controls, respectively. Supplementary Figure 3. A641T mutant does not activate RET kinase. (A) NIH3T3 cells were transduced stably with EV, RET WT or mutants (A641T and C634R). The lysates were analyzed by western blotting with anti-phospho RET (Y905) and anti-RET antibodies. (B) Bar graphs demonstrated the quantification of western blotting bands in sFigure 3A, normalized to WT control. (C) A641T mutant is not able to increase the cell viability of NIH3T3 cells. NIH3T3 cells stably expressing EV, RET WT, A641T, and C634R (positive control) were cultured on 96-well plates and the viability was measured by CTG assay at day 2 .
\end{abstract}

Additional file 2: Supplementary Table 1. Detailed genomic information of 100 PTKs genes and the mutation frequency in 605 EOC patients from TCGA. Supplementary Table 2. List of RET missense mutations identified in EOC patients from the TCGA. Supplementary Table 3. Summary of all RET missense mutations identified in EOC patients.

\section{Abbreviations}

PTKs: Protein tyrosine kinases; EOC: Epithelial ovarian cancer; PARPis: Poly ADP-ribose polymerase inhibitors; VEGF: Vascular endothelial growth factor; EGFR: Epidermal growth factor receptor; GDNF: Glial cell line-derived neurotrophic factor; NSCLC: Non-small-cell lung cancer; MTC: Medullary thyroid carcinoma; FDA: Food and Drug Administration; PFS: Progression-free survival; OS: Overall survival; TCGA: The Cancer Genome Atlas;

COSMIC: Catalogue of Somatic Mutations in Cancer; ICGC: International 
Cancer Genome Consortium; CCLE: Broad Institute Cancer Cell Line Encyclopedia

\section{Acknowledgments}

The authors are grateful to Professor Lois M. Mulligan (Division of Cancer Biology and Genetics, Department of Pathology and Molecular Medicine, Cancer Research Institute, Queen's University, Kingston, Canada) for pCDH3.1RET51-WT plasmid.

\section{Authors' contributions}

YXW and YL: study concept and design. LYG, ZL, FFX, YZP, CYZ, HST, HZ, CC, YYZ, TZ, HYJ, XNJ, YXW and YL: acquisition of data. LYG, ZL, FFX, YZP, CYZ, $H S T, H Z, C C, Y Y Z, T Z, H Y J, X N J, Y X W$ and YL: analysis and interpretation of data. LYG, YXW and YL: writing the manuscript. The author(s) read and approved the final manuscript.

\section{Funding}

This work was supported by grants from the Natural Science Foundation of Shanghai (grant numbers: 19ZR1407100), and the National Natural Science Foundation of China (81572642).

\section{Availability of data and materials}

All data generated or analyzed during this study are included in this published article and its supplementary information file. Further details are available from the corresponding author on reasonable request.

\section{Ethics approval and consent to participate}

The animal experiments were approved by the institutional animal care and use committee of the Shanghai Institutes for Biological Sciences, Chinese Academy of Science.

\section{Consent for publication}

All authors give consent for the publication of the manuscript.

\section{Competing interests}

The authors declare that they have no competing interests.

\begin{abstract}
Author details
'Department of Gynecology, Obstetrics and Gynecology Hospital, Fudan University Shanghai, 419 Fangxie Rd, Shanghai 200011, People's Republic of China. ${ }^{2}$ Key Laboratory of Tissue Microenvironment and Tumor, SINH Changzheng Hospital Joint Center for Translational Medicine, Institutes for Translational Medicine (CAS-SMMU), Shanghai Institute of Nutrition and Health, University of Chinese Academy of Sciences, Chinese Academy of Sciences, 320 Yueyang Rd, Shanghai 200031, People's Republic of China. ${ }^{3}$ Department of Pathology, Obstetrics and Gynecology Hospital, Fudan University Shanghai, Shanghai, People's Republic of China.
\end{abstract}

Received: 28 November 2019 Accepted: 8 March 2020

Published online: 23 March 2020

\section{References}

1. Siegel RL, Miller KD, Jemal A. Cancer statistics, 2019. CA Cancer J Clin. 2019; 69(1):7-34.

2. Neijt JP, Ten BHW, van der Burg ME, et al. Randomised trial comparing two combination chemotherapy regimens (Hexa-CAF vs CHAP-5) in advanced ovarian carcinoma. Lancet. 1984;2(8403):594-600.

3. Pujade-Lauraine E, Combe P. Recurrent ovarian cancer. Ann Oncol. 2016; 27(Suppl 1):i63-5.

4. Lheureux S, Braunstein M, Oza AM. Epithelial ovarian cancer: evolution of management in the era of precision medicine. CA Cancer J Clin. 2019;69(4): 280-304.

5. Ledermann JA, Harter P, Gourley C, et al. Overall survival in patients with platinum-sensitive recurrent serous ovarian cancer receiving olaparib maintenance monotherapy: an updated analysis from a randomised, placebo-controlled, double-blind, phase 2 trial. Lancet Oncol. 2016;17(11): 1579-89.

6. Monk BJ, Minion LE, Coleman RL. Anti-angiogenic agents in ovarian cancer: past, present, and future. Ann Oncol. 2016;27(Suppl 1):i33-9.

7. Wu P, Nielsen TE, Clausen MH. FDA-approved small-molecule kinase inhibitors. Trends Pharmacol Sci. 2015;36(7):422-39.
8. Inoue A, Kobayashi K, Maemondo $\mathrm{M}$, et al. Updated overall survival results from a randomized phase III trial comparing gefitinib with carboplatinpaclitaxel for chemo-naive non-small cell lung cancer with sensitive EGFR gene mutations (NEJ002). Ann Oncol. 2013;24(1):54-9.

9. Mulligan LM. RET revisited: expanding the oncogenic portfolio. Nat Rev Cancer. 2014;14(3):173-86.

10. Donis-Keller H, Dou S, Chi D, et al. Mutations in the RET proto-oncogene are associated with MEN 2A and FMTC. Hum Mol Genet. 1993;2(7):851-6.

11. Hofstra RM, Landsvater RM, Ceccherini I, et al. A mutation in the RET protooncogene associated with multiple endocrine neoplasia type $2 \mathrm{~B}$ and sporadic medullary thyroid carcinoma. Nature. 1994;367(6461):375-6.

12. Romei C, Elisei R. RET/PTC translocations and clinico-pathological features in human papillary thyroid carcinoma. Front Endocrinol (Lausanne). 2012;3:54.

13. Kohno T, Ichikawa H, Totoki Y, et al. KIF5B-RET fusions in lung adenocarcinoma. Nat Med. 2012;18(3):375-7.

14. Romei C, Ciampi R, Elisei R. A comprehensive overview of the role of the RET proto-oncogene in thyroid carcinoma. Nat Rev Endocrinol. 2016;12(4): 192-202.

15. Wells SJ, Robinson BG, Gagel RF, et al. Vandetanib in patients with locally advanced or metastatic medullary thyroid cancer: a randomized, doubleblind phase III trial. J Clin Oncol. 2012;30(2):134-41.

16. Torre LA, Trabert B, DeSantis CE, et al. Ovarian cancer statistics, 2018. CA Cancer J Clin. 2018;68(4):284-96.

17. Li Z, Shao C, Liu X, et al. Oncogenic ERBB2 aberrations and KRAS mutations cooperate to promote pancreatic ductal adenocarcinoma progression. Carcinogenesis. 2019. https://doi.org/10.1093/carcin/bgz086.

18. Adzhubei IA, Schmidt S, Peshkin L, et al. A method and server for predicting damaging missense mutations. Nat Methods. 2010;7(4):248-9.

19. Kumar P, Henikoff S, Ng PC. Predicting the effects of coding nonsynonymous variants on protein function using the SIFT algorithm. Nat Protoc. 2009;4(7):1073-81.

20. Ceccherini I, Pasini B, Pacini F, et al. Somatic in frame deletions not involving juxtamembranous cysteine residues strongly activate the RET proto-oncogene. Oncogene. 1997;14(21):2609-12.

21. Borrello MG, Aiello A, Peissel B, et al. Functional characterization of the MTCassociated germline RET-K666E mutation: evidence of oncogenic potential enhanced by the G691S polymorphism. Endocr Relat Cancer. 2011;18(4): 519-27.

22. Arts FA, Chand D, Pecquet C, et al. PDGFRB mutants found in patients with familial infantile myofibromatosis or overgrowth syndrome are oncogenic and sensitive to imatinib. Oncogene. 2016;35(25):3239-48.

23. Cheng F, Liang H, Butte AJ, Eng C, Nussinov R. Personal mutanomes meet modern oncology drug discovery and precision health. Pharmacol Rev. 2019;71(1):1-19.

24. Ledermann JA. Front-line therapy of advanced ovarian cancer: new approaches. Ann Oncol. 2017;28(suppl_8):viii46-50.

25. Pegram MD, Pauletti G, Slamon DJ. HER-2/neu as a predictive marker of response to breast cancer therapy. Breast Cancer Res Treat. 1998;52(1-3):65-77.

26. Deininger $M$, Buchdunger $E$, Druker $B J$. The development of imatinib as a therapeutic agent for chronic myeloid leukemia. Blood. 2005;105(7):2640-53.

27. Wells SJ, Asa SL, Dralle H, et al. Revised American Thyroid Association guidelines for the management of medullary thyroid carcinoma. Thyroid. 2015:25(6):567-610

28. Plaza-Menacho I, Barnouin K, Goodman K, et al. Oncogenic RET kinase domain mutations perturb the autophosphorylation trajectory by enhancing substrate presentation in trans. Mol Cell. 2014;53(5):738-51.

29. Gujral TS, Singh VK, Jia Z, Mulligan LM. Molecular mechanisms of RET receptor-mediated oncogenesis in multiple endocrine neoplasia 2B. Cancer Res. 2006:66(22):10741-9.

30. Sawai H, Okada Y, Kazanjian K, et al. The G691S RET polymorphism increases glial cell line-derived neurotrophic factor-induced pancreatic cancer cell invasion by amplifying mitogen-activated protein kinase signaling. Cancer Res. 2005;65(24):11536-44.

31. Narita N, Tanemura A, Murali R, et al. Functional RET G691S polymorphism in cutaneous malignant melanoma. Oncogene. 2009;28(34):3058-68.

32. BLU-667 targets RET-altered cancers. Cancer Discov. 2018;8(6):OF8. https:// doi.org/10.1158/2159-8290.CD-NB2018-050.

33. Subbiah $\vee$, Velcheti $V$, Tuch $B B$, et al. Selective RET kinase inhibition for patients with RET-altered cancers. Ann Oncol. 2018;29(8):1869-76.

34. Subbiah V, Gainor JF, Rahal R, et al. Precision targeted therapy with BLU-667 for RET-driven cancers. Cancer Discov. 2018;8(7):836-49. 
35. BLU-667 controls RET-altered thyroid cancers. Cancer Discov. 2019;9(9):OF5. https://doi.org/10.1158/2159-8290.CD-NB2019-084.

36. Bronte G, Ulivi P, Verlicchi A, Cravero P, Delmonte A, Crino L. Targeting RETrearranged non-small-cell lung cancer: future prospects. Lung Cancer (Auckl). 2019;10:27-36.

37. Sheng Q, Liu J. The therapeutic potential of targeting the EGFR family in epithelial ovarian cancer. Br J Cancer. 2011;104(8):1241-5.

38. Dancey J. Targeted therapies and clinical trials in ovarian cancer. Ann Oncol. 2013;24(Suppl 10):x59-63.

39. Wang Y, Li L, Douville C, et al. Evaluation of liquid from the Papanicolaou test and other liquid biopsies for the detection of endometrial and ovarian cancers. Sci Transl Med. 2018;10(433). https://doi.org/10.1126/scitranslmed.aap8793.

40. Phallen J, Sausen M, Adleff V, et al. Direct detection of early-stage cancers using circulating tumor DNA. Sci Transl Med. 2017;9(403). https://doi.org/10. 1126/scitransImed.aan2415.

\section{Publisher's Note}

Springer Nature remains neutral with regard to jurisdictional claims in published maps and institutional affiliations.

Ready to submit your research? Choose BMC and benefit from:

- fast, convenient online submission

- thorough peer review by experienced researchers in your field

- rapid publication on acceptance

- support for research data, including large and complex data types

- gold Open Access which fosters wider collaboration and increased citations

- maximum visibility for your research: over $100 \mathrm{M}$ website views per year

At BMC, research is always in progress.

Learn more biomedcentral.com/submissions 